\title{
DEVELOPMENT OF MOTOR INNERVATION OF THE CHICK FOLLOWING DORSAL-VENTRAL LIMB BUD ROTATIONS ${ }^{1}$
}

\author{
BETTY ALICE FERGUSON \\ Department of Biology, B-022, University of California, San Diego, La Jolla, California 92093 \\ Received October 19, 1982; Revised March 30, 1983; Accepted March 31, 1983
}

\begin{abstract}
To test mechanisms which motoneurons may use to grow to their appropriate targets, I rotated the limb around the dorsal-ventral axis prior to motoneuron outgrowth. The positions of motoneurons in the spinal cord innervating individual muscles and muscle masses were then determined using retrograde horseradish peroxidase uptake.

Motoneurons innervated their appropriate muscles after dorsal-ventral limb rotation, before and after motoneuron death. Thus, cell death does not serve to remove errors in matching between motor nuclei and their corresponding muscles after dorsoventral rotation of the limb. Motoneurons must be specified for a peripheral target prior to outgrowth, and they grow to that target relatively directly.

Axons compensated for the limb rotation by first collecting into groups in a position appropriate for the normal limb orientation, then shifting dorsal-ventral position within the plexus and proximal nerve trunk. Based on these results it is hypothesized that axons destined to innervate dorsal or ventral musculature might use chemospecific cues during growth to maintain appropriate positions within the nerve with respect to limb orientation.
\end{abstract}

In the chick spinal cord, as in several other vertebrate species (Romanes, 1951, 1964; Sharrard, 1955; Cruce, 1974; Bixby et al., 1980), all of the neurons that innervate one particular muscle are grouped into a single nucleus which is found in a characteristic position in the lateral motor column (Landmesser, 1978a). The position of a motoneuron within the spinal cord is related to the embryonic origin of the muscle it innervates (Landmesser, 1978a; Hollyday, 1980). Within the developing limb, the musculature initially exists as two muscle masses, dorsal and ventral, which cleave to form individual muscles (Romer, 1927; Wortham, 1948). Motoneurons innervating muscles derived from the embryonic ventral muscle mass are located medially in the transverse axis of the spinal cord, whereas motoneurons innervating muscles derived from the embryonic dorsal muscle mass are located laterally. There is a strict relationship between motoneuron position in the transverse axis of the spinal cord and the embryonic origin of the muscle it innervates. This pattern of motoneuron projection to the limb sug-

\footnotetext{
${ }^{1}$ This work was part of a dissertation submitted in partial fulfillment of the requirements for the Ph.D. degree at Yale University. I thank Marcia Honig, Cynthia Lance-Jones, and in particular, Lynn Landmesser, for their support. I thank Christine Holt, John Bixby, and Bill Harris for suggestions concerning the manuscript. This work was supported by National Institutes of Health Grant NS10666 to Lynn T. Landmesser.
}

gests that there could be position-dependent axon outgrowth into the limb during development. Motoneurons could be specified for a particular peripheral termination by virtue of their position in the spinal cord, or motoneuron axons could maintain the same relative positions during outgrowth as those of their somas, a mechanism not requiring motoneuron specification (Horder, 1978).

If the location of the target of a motoneuron axon is changed, for example by rotation of the limb bud early in development, the resulting pattern of limb innervation could suggest what kinds of cues motoneuron axons use to find the targets. If axons follow chemospecific labels that either mark the path to their target or emanate from that target (Sperry, 1963), motoneuron axons should be able to detect and perhaps compensate for changes in the position of their target. If motoneuron axon growth is nonselective for a particular target and is due, for example, to selective adhesion (Ludueña and Wessels, 1973; Letourneau, 1975a, b), glial cell orientation (Rakic, 1971; Suburo et al., 1979), or specific growth through intercellular spaces (Lyser, 1971; Silver and Robb, 1979; Silver and Sidman, 1980), any change in the environment through which they grow will channel axons to an abnormal target. If axons simply synapse with the first uninnervated territory that they encounter, altering the sequence in which they encounter muscles will also alter the pattern of limb innervation.

To determine what kind(s) of cues motor axons do use, 
I changed the environment into which they grew by rotating the limb in such a way as to exchange the positions of the dorsal and ventral musculature. There is a clear separation in the transverse plane of the spinal cord of the positions of motoneurons innervating the dorsal and ventral musculature of the limb; thus the results of this manipulation were easily assessed using retrograde uptake of horseradish peroxidase (HRP) to localize the positions of motoneurons within the spinal cord innervating particular muscles or muscle masses. The result is that motoneurons innervate their normal targets after dorsal-ventral limb rotation, both before and after the period of naturally occurring cell death (Hamburger, 1975). Axons compensated for the limb rotation by altering their positions within the plexus and proximal nerve trunk, prior to reaching their target muscles. These results support those of Lance-Jones and Landmesser (Lance-Jones and Landmesser, 1980; Landmesser and Lance-Jones, 1981) following embryonic rotations and displacements in the anterior-posterior axis of the chick spinal cord and limb.

\section{Materials and Methods}

Retrograde HRP labeling and processing. White Leghorn chick embryos of stages 28 to 36 (51/2 to 11 days; Hamburger and Hamilton, 1951) were decapitated, eviscerated, and placed in oxygenated Tyrode solution containing (mM): $\mathrm{NaCl}, 150 ; \mathrm{KCl}, 3 ; \mathrm{CaCl}_{2}, 3 ; \mathrm{MgCl}_{2}, 1$; Tris, 10 ; glucose, $12 ; \mathrm{pH} 7.4$ at room temperature $\left(20\right.$ to $\left.22^{\circ} \mathrm{C}\right)$. To ensure oxygenation of the spinal cord, a ventral laminectomy was done extending from the first brachial through the last lumbosacral (LS) segments.

In experiments involving identification of individual muscles, the skin was removed from the limb. Usually, both limbs of an embryo were injected with HRP.

HRP dissolved in Tyrode solution $(100 \mathrm{mg} / \mathrm{ml})$ was pressure injected into the muscle (stages 34 to 36 ) or whole muscle mass (stages 28 to 31 ) through glass pipettes with tip diameters of 30 to $100 \mu \mathrm{m}$. Embryos were then incubated for $5 \mathrm{hr}$ at $32^{\circ} \mathrm{C}$ according to the technique of Landmesser (1978a). After fixation in $2 \%$ glutaraldehyde at $4^{\circ} \mathrm{C}$ overnight, embryos were washed repeatedly in Tris buffer ( $\mathrm{pH}$ 7.2) for over a week with a final 4-hr rinse of $0.1 \mathrm{M}$ sodium borohydrate in Tris buffer, then incubated for $2 \mathrm{hr}$ in diaminobenzidine (10 $\mathrm{mg} / 10 \mathrm{ml}$ of Tris) at $4^{\circ} \mathrm{C}$, and allowed to continue incubation in the diaminobenzidine at room temperature (20 to $22^{\circ} \mathrm{C}$ ) for $5 \mathrm{hr}$ with the addition of $0.03 \%$ hydrogen peroxide. The embryos were then dehydrated in a graded series of alcohols and embedded in paraffin.

Spinal cords and limbs of embryos at stages 33 to 36 were sectioned separately, transverse to their long axis, at $10 \mu \mathrm{m}$. Embryos of stages 28 to 31 were processed in two ways. Initially, whole embryos of these stages were simply sectioned at $10 \mu \mathrm{m}$ either transversely or parallel to the spinal cord, depending on whether localization of motoneurons within the cord or reconstruction of nerve patterns in the limb was intended. In order to determine both the location of motoneurons and the paths of axon growth within a single embryo, limbs were sectioned transversely in whole embryos until the dorsal root ganglia were reached. Both the dorsoventrally rotated and control limbs were sectioned in this way. The spinal cord was then reimbedded and sectioned transversely to localize HRP-stained motoneurons. Sections were counterstained with cresyl violet.

In order to reconstruct axon pathways from the cord through the limb, motoneuron axons must stain for HRP throughout their length. Not all preparations showed this type of staining and thus could not be used for study. Diffuse staining of axons seemed to correlate with a higher degree of damage to motor axon terminals.

The HRP injection site was confirmed by inspection of the spread of HRP reaction product in histological sections of the limb. Any embryo in which the reaction produce was not strictly localized in the intended muscle or muscle mass was excluded from further study.

The number of HRP-stained motoneurons was counted using a Zeiss Universal microscope at $\times 500$ magnification (stages 34 to 36 ) or $\times 800$ magnification to check light staining and for embryos of stages 28 to 31 . Histograms were made of motoneuron locations and numbers by counting neurons in every third section (stages 34 to 36 ) or every second section (stages 28 to 31). Each histogram consists of motoneuron counts for three or four embryos normalized by alignment at the first section of LS segment 1, defined as beginning midway between the last thoracic and first LS dorsal root ganglia and ending midway between LS dorsal root ganglia 1 and 2. The composite histograms were expressed as the mean percentage of total labeled cells per 20 or 30 $\mu \mathrm{m} \pm \mathrm{SE}$, depending upon the stage. No correction was used for double counting.

Motor nuclei were localized in the transverse plane of the spinal cord by making camera lucida drawings at a magnification of $\times 500$ of every stained neuron in every second (stages 28 to 31 ) or third (stages 33 to 36 ) section. Drawings were combined to produce a composite representation of motoneuron positions within the spinal cord. Sections were aligned using the central canal and white matter as reference points.

Embryonic limb bud rotations. 'These operations were done at stages 16 to 17 , when fewer than $3 \%$ of shank motoneurons have stopped dividing (Hollyday and Hamburger, 1977) and no motoneurons have extended axons into the limb. The embryos were candled, their air sacs were pierced to allow the embryos to sink and stabilize in position, and the shells were opened with a dental drill. A tear was made in the vitelline membrane; the embryo was dampened with Tyrode solution and then was stained with sterile $2 \%$ neutral red in saline as an aid to visualization.

To achieve purely dorsal-ventral limb bud rotations, limb buds were exchanged between the right and left sides of two embryos of the same stage after rotating the limb buds dorsoventrally, leaving the anterior-posterior axis normal. Limb buds were excised using fire-sharpened tungsten needles and exchanged using a Spemann pipette. Operated embryos were not moved for $1 / 2 \mathrm{hr}$ after operation to allow the limb bud to heal after replacement on the body. After operation, eggs were resealed with glass coverslips and paraffin and were returned to the incubator to continue development until stages 28 to 36 .

Commonly, embryos inspected at a later stage were limbless or had malformed limbs. Approximately $5 \%$ of all operations were successful, but a success rate of $75 \%$ 
CONTROL

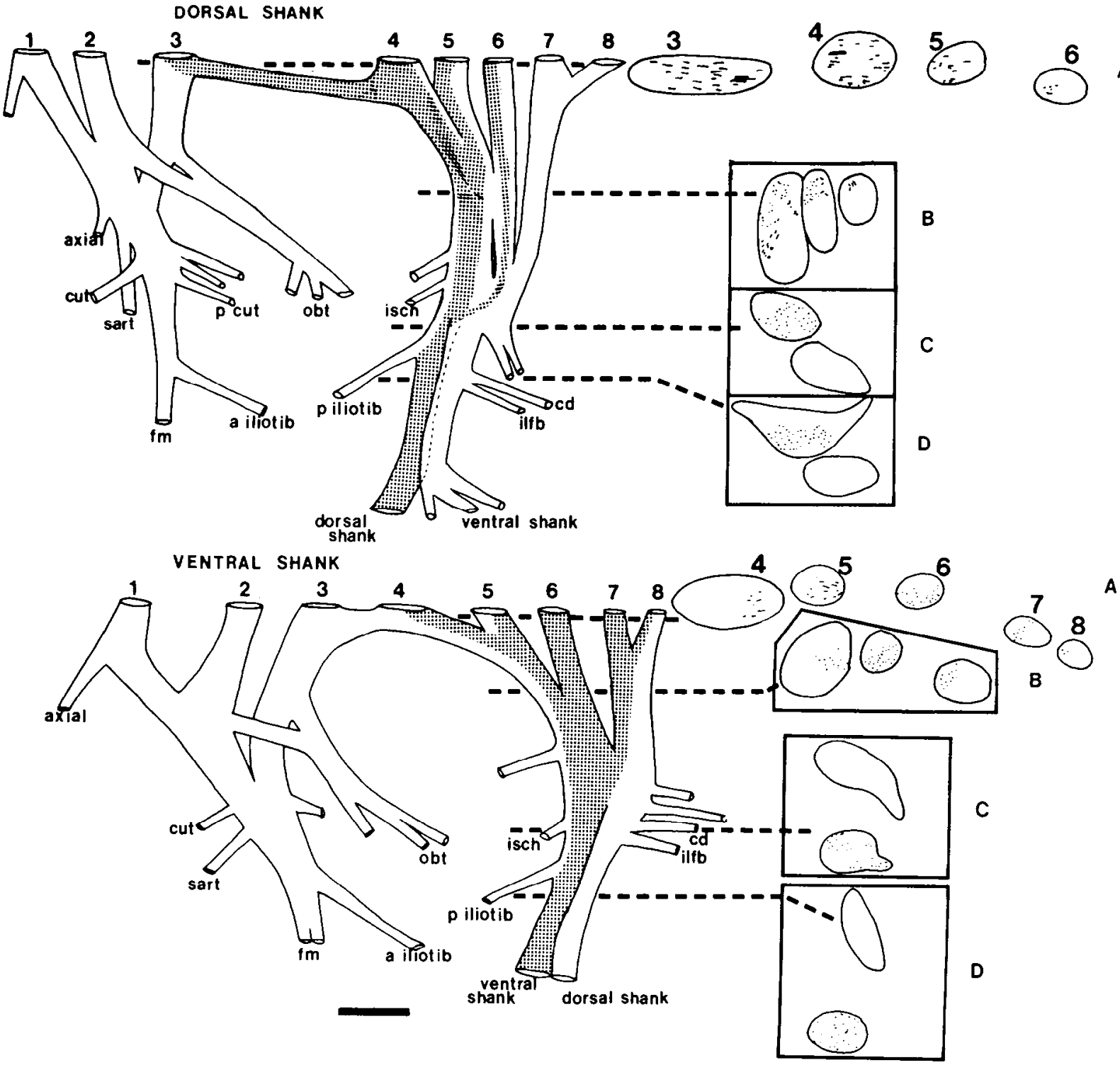

Figure 1. Medial view reconstructions of the nerve patterns in the hindlimb of two control stage 29 embryos. Numbers identify the spinal nerves in each reconstruction. In the top reconstruction, the dorsal muscle mass was injected with HRP; staining was found in spinal nerves 3 to 6 , shown by stippling. 'The bottom reconstruction shows HRP staining after injection of the shank ventral muscle mass with HRP. Staining (stippling) is seen in spinal nerves 4 to 8 , thus spinal nerves 4 to 6 innervate both the dorsal and ventral shank musculature. However, transverse sections of the nerves show the axons to dorsal and ventral musculature separate from each other in the medial-lateral axis during outgrowth. The insets $(A$ to $D)$ are camera lucida drawings of transverse sections from which the reconstructions were made; dashed lines indicate the level. Lateral is up; medial is down. Insets of the top reconstruction show that stained axons to dorsal musculature, initially intermingled with unstained axons (inset $A$ ), then collect laterally (up) in inset $B$; axons to ventral musculature collect relatively medially (down) in inset $B$ of the bottom reconstruction. The axons synapse with the shank muscles distal to the point at which the reconstructions end. In both reconstructions, nerves to the dorsal and ventral shanks are shown adjacent for clarity; in reality, insets $D$ show that the nerve to the ventral shank is directly medial, overlying the nerve to the dorsal shank. cut, anterior cutaneous; $f m$, femorotibialis; $a$ iliotib, anterior iliotibialis; sart, sartorius; obt, obturator; $p$ cut, posterior cutaneous; isch, ischioflexorius; $c d$, caudilioflexorius; $i l f b$, iliofibularis; $p$ iliotib, posterior iliotibialis. Calibration bar, $200 \mu \mathrm{m}$.

was achieved in the latest experiments. Embryos with malformed limbs were discarded.

\section{Results}

Axon pathways in normal embryos. Since a given spinal root contains motoneuron axons destined for both ventral and dorsal muscle masses (Landmesser and Morris, 1975), and since axons project appropriately from early stages prior to motoneuron death (Landmesser, 1978b), it is clear that axons innervating the ventral and dorsal shanks must separate from one another at some point in their growth. This separation could occur at any point in their growth, either immediately upon exiting the spinal cord, or upon encountering the muscles themselves. To determine at what point motoneurons innervating the ventral and dorsal shanks separate, ventral or 
dorsal shanks of stage 29 embryos were injected with HRP. In some embryos, this results in diffuse HRP staining of motoneuron axons. The embryos were then sectioned transverse to the limb, and reconstructions were made of the paths of the stained axons from their point of exit from the spinal cord to their terminations.

Figure 1 shows reconstructions in which axons projecting to the ventral shank were labeled in one embryo and those projecting to the dorsal shank were labeled in another embryo. The insets are camera lucida tracings of transverse sections at the levels in outgrowth indicated by the dotted lines and show the positions of HRP-labeled axons within the nerves. Axons destined for either dorsal or ventral shank aggregate and take up characteristic positions with respect to the dorsal-ventral axis of the embryo even before the spinal nerves converge to form the sciatic plexus (insets $B$ of both reconstructions). Axons that innervate the dorsal shank collect laterally (up in inset $B$, top reconstruction), whereas axons innervating the ventral shank collect medially (down in inset $C$, bottom reconstruction) in their growth to the periphery.

Projection patterns following dorsal-ventral rotations of the limb bud. In 10 embryos in which the limb was rotated dorsoventrally at stages 16 to 17 , localization of the motor nuclei following the period of motoneuron death showed that motoneurons projected to their original muscle mass, even though its position had been altered with respect to the dorsal-ventral axis of the embryo. Figure 2 shows the typical appearance of HRP-stained motoneurons within the spinal cord of a control embryo following injection of the enzyme into two different muscles. Note that motoneurons innervating the tibialis anterior (Fig. 2, left), a muscle derived from the embryonic dorsal muscle mass, are found more laterally within the motor column than are the motoneurons innervating the medial gastrocnemius (Fig. 2, right), originating from the ventral muscle mass.

Projections to shank muscles. Figure 3 shows an example of motoneuron locations in the spinal cord following injection of HRP into the dorsal shank of a dorsoventrally rotated limb and, for comparison, the motoneuron locations following injection of HRP into the dorsal shank of a control limb. The locations of dorsal shankinnervating motoneurons within the mediolateral plane of the spinal cord are the same in control embryos and

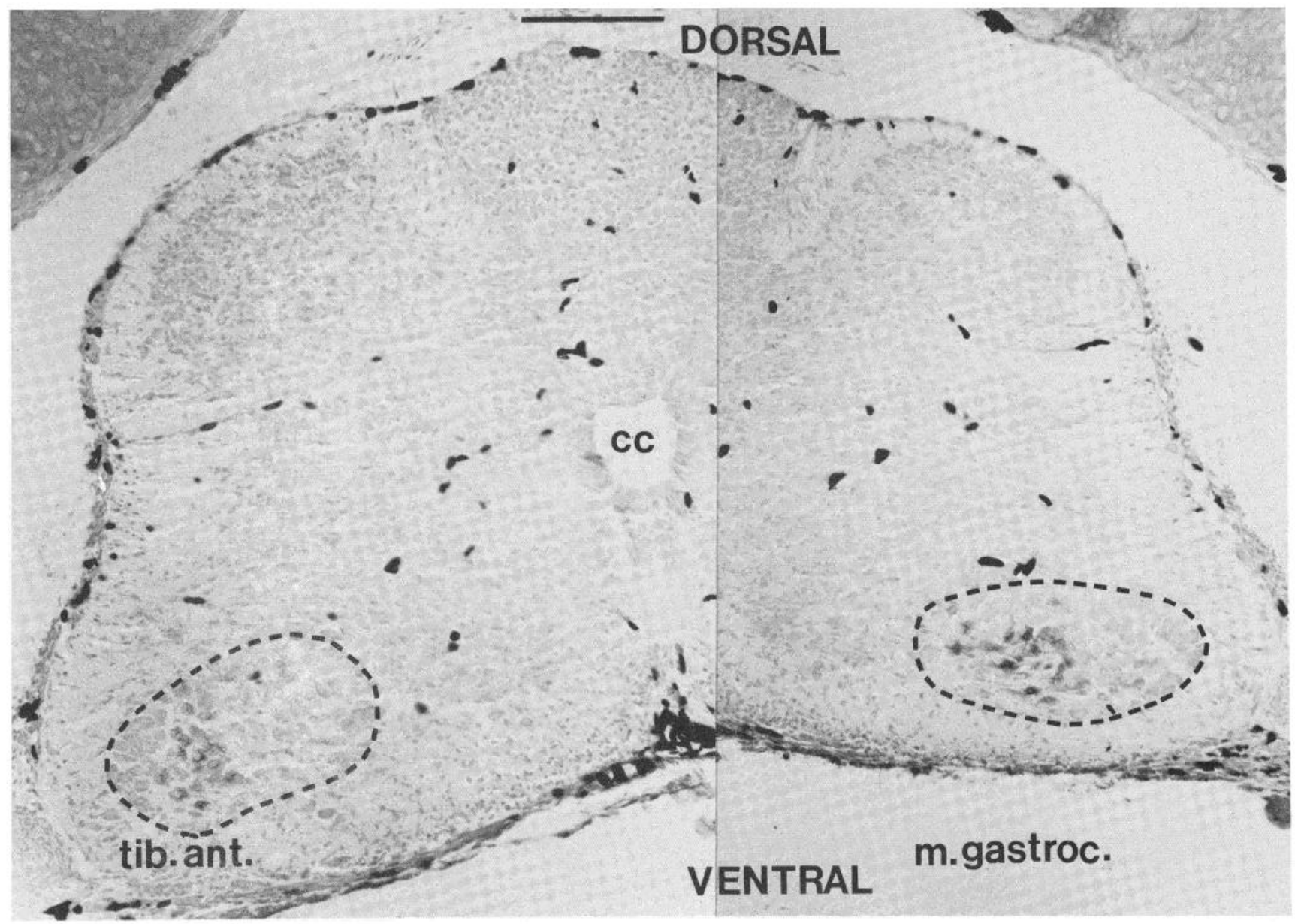

Figure 2. Photomicrograph of transverse sections through a normal stage 36 chick spinal cord in LS4 showing typical distribution and appearance of stained motoneurons after injection of HRP into the medial gastrocnemius (right) and the tibialis anterior (left). Dashed outlines indicate the extent of the entire lateral motor column, within which are stained motoneurons. Note that the medial gastrocnemius ( $m$. gastroc.) motoneurons are in a relatively more medial position in the lateral motor column than are the motoneurons innervating the tibialis anterior (tib. ant.). Pictures of the right and left sides of the spinal cord are taken from the same embryo $30 \mu \mathrm{m}$ apart. Calibration bar, $100 \mu \mathrm{m}$. 
embryos with dorsoventrally rotated limbs, in a lateral position. This result was found in four of four embryos.

Motoneurons projecting to the ventral shank were also localized appropriately; i.e., in a relatively medial position within the spinal cord. This is shown in Figure 4, where the positions of motoneurons innervating the ventral shank in a control embryo are similar to those in an embryo with a dorsoventrally rotated limb. This result was obtained in three of three embryos.

Motoneurons were also located in appropriate rostrocaudal positions. However, since there is normally considerable overlap in the distributions of dorsally and ventrally projecting motoneurons along this axis, this result is not as meaningful as the data just presented.
VENTRAL SHANK
CONTROL

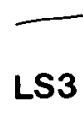

DORSAL SHANK

CONTROL

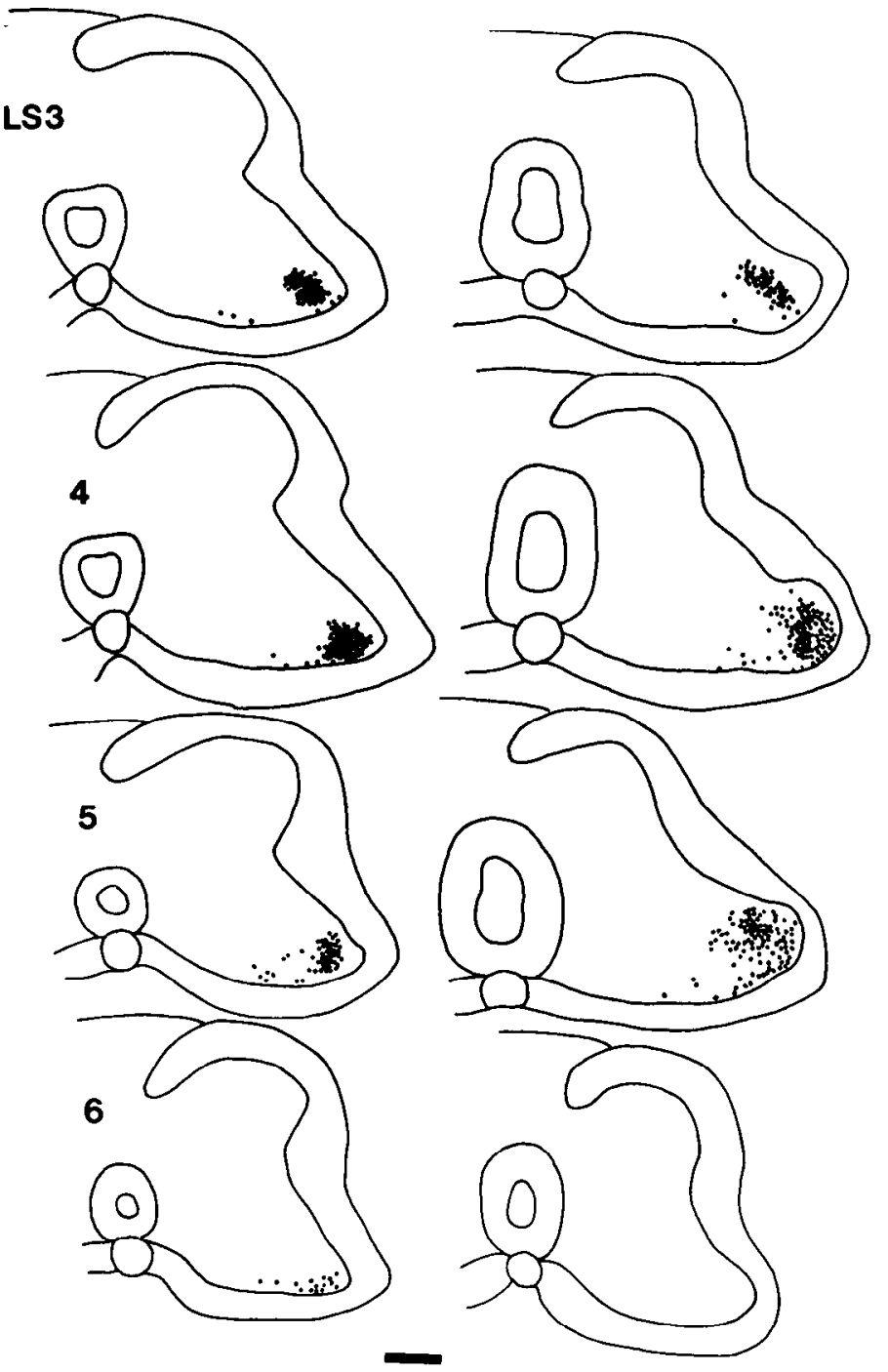

3

\section{ROTATION}

Figures 3 and 4. Positions of stained motoneurons within the transverse plane of the spinal cord following injections of HRP into muscles of the dorsal shank (Fig. 3) and ventral shank (Fig. 4) in control (left) and experimental (right) embryos at stage 36 . Each cross-section shows all stained motoneurons within a LS segment in every third 10- $\mu \mathrm{m}$ section. Each stained motoneuron is indicated by a dol. Calibration bar, $100 \mu \mathrm{m}$. 
The histograms of Figure 5 indicate the distributions of motoneurons along the rostrocaudal axis of the spinal cord innervating the ventral shank (Fig. $5 A$ ) and dorsal shank (Fig. 5B) in control embryos and embryos with dorsal-ventral limb rotations. Although the variation in segmental contribution to a muscle mass was larger in animals with dorsoventrally rotated limbs, it is clear that motoneurons innervating a muscle mass after dorsalventral limb rotation were appropriate in both their rostrocaudal and mediolateral positions within the spinal cord.

Projections to thigh muscles. To determine whether similar results would be found for muscles located more proximally in the limb, two thigh muscles were injected in embryos with dorsal-ventral limb rotations. In the example shown in Figure 6, motoneurons innervating the sartorius muscle were localized following dorsal-ventral limb rotation. Comparison of the control and experimental sartorius nuclei shows that the motoneurons are located in the appropriate segments (Fig. 6B) and the appropriate mediolateral positions within each segment (Fig. 6A). This also is true of motoneurons innervating the iliofibularis muscle after dorsal-ventral limb rotation. The motoneurons are located in their normal positions, both in the rostrocaudal (Fig. $7 B$ ) and mediolateral (Fig. $7 A$ ) spinal cord axes. Thus, even for muscles located in a very proximal limb position whose motoneurons would have had a minimal distance to detect and respond to the altered limb orientation, motoneurons still connected specifically with their normal target.

In every embryo with a dorsoventrally rotated limb, those motoneurons innervating a muscle (sartorius, 2 cases; iliofibularis, 1 case) or muscle mass (dorsal muscle mass of shank, 4 cases; ventral muscle mass of shank, 3 cases) were from appropriate positions in the spinal cord.

Although the motoneurons innervating a muscle or muscle mass after dorsal-ventral limb rotation were always appropriate, commonly, an entire lumbosacral segment which normally would innervate a muscle did not do so in embryos with dorsoventrally rotated limbs. There are several possible reasons for this. In some cases, it was found that in an attempt to inject HRP into the entire dorsal or ventral muscle mass, the injection obviously did not fill the entire muscle mass. This also occurred with control injections. In addition, it was apparent that in some embryos a spinal root did not enter normally into the crural or sciatic plexus, but instead branched to the tail or body wall, or ended in a neuroma. This was probably due to unintended slight shifts of the repositioned limb bud along the anterior-posterior axis. Similar results have been found when the limb was intentionally shifted varying distances along this axis (Landmesser and Lance-Jones, 1981). Most commonly, when there was a deficit in segmental contribution to the limb, spinal nerve 1 did not enter the limb, spinal nerve 3 did not send a branch to the sciatic, or spinal nerves 7

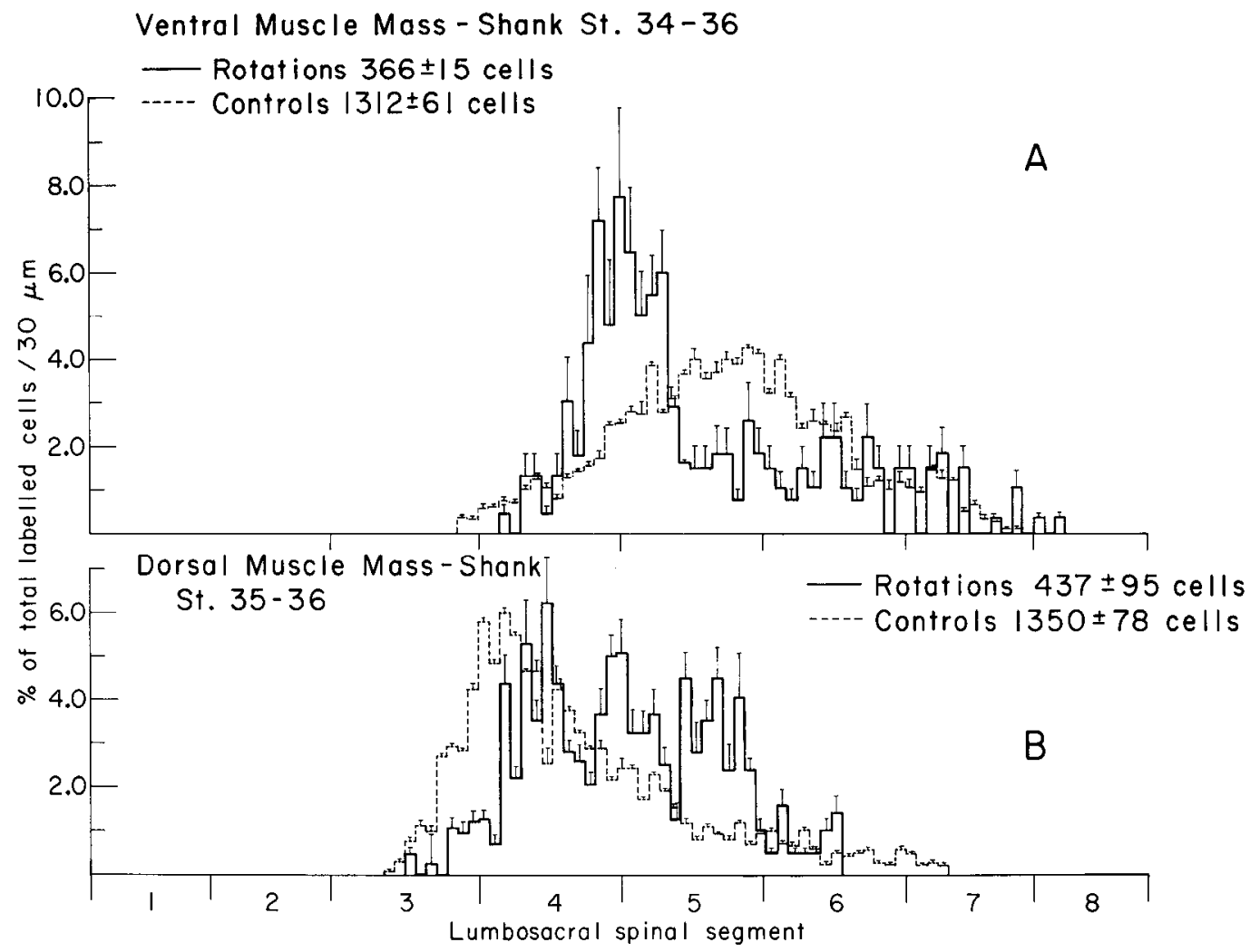

Figure 5. Rostrocaudal position of motoneurons innervating the ventral muscle mass of the shank in control and experimental embryos at stages 34 to $36(A)$, and the dorsal muscle mass of the shank in control and experimental embryos at stages 35 to $36(B)$. Each histogram was pooled from three embryos showing mean percentage of each nucleus occurring in successive $30-\mu \mathrm{m}$ increments along the lumbosacral cord. The mean number of labeled cells per nucleus $\pm \mathrm{SE}$ is also shown. Error bars drawn in only one direction represent SE. 
and/or 8 did not enter the sciatic, but instead sent large branches to the tail.

Projection patterns prior to the motoneuron cell death period following dorsal-ventral rotations of the limb bud. The above results show that motoneurons innervate their original muscles at stages 34 to 36 following dorsalventral rotation of the hindlimb. However, since stages 34 to 36 are after the main period of motoneuron death in the chick (Hamburger, 1975), death of inappropriately connecting motoneurons might account for this apparent specificity in axonal growth. For this reason, the positions of motoneurons innervating either the entire ventral shank or the dorsal shank were localized in eight

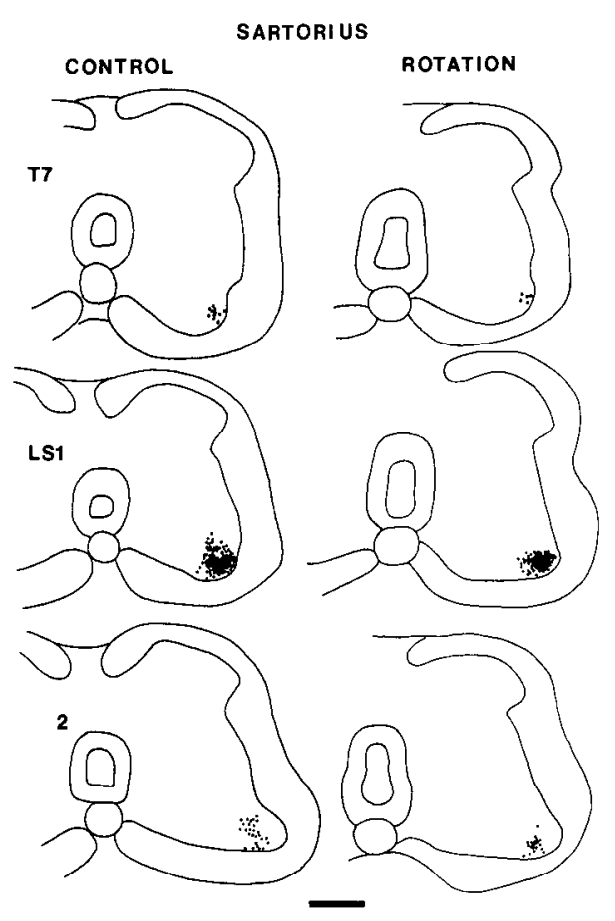

A

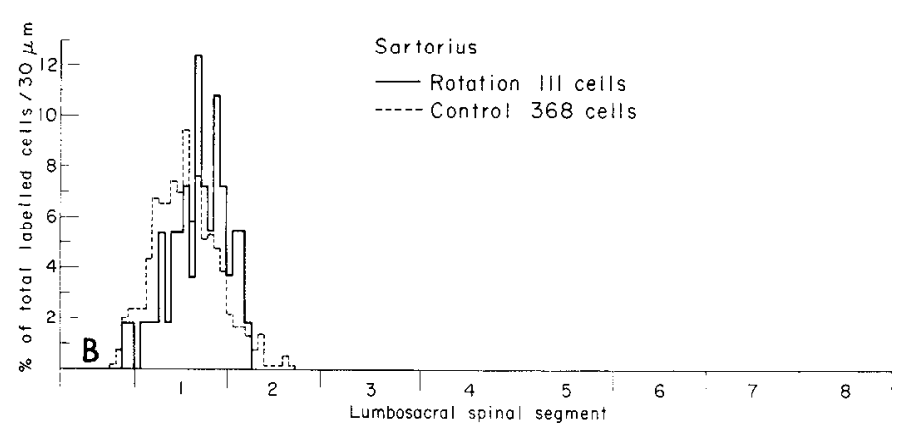

Figure 6. A, Transverse sections showing stained motoneurons following injections of HRP into the sartorius muscle in control (left) and experimental (right) embryos at stage 36 . Each cross-section pools all stained motoneurons (indicated by dots) in every third $10-\mu \mathrm{m}$ section within a LS segment. Calibration bar, $100 \mu \mathrm{m}$. B, Rostrocaudal position motoneurons innervating the sartorius muscle in stage 36 control and experimental embryos. Histograms show the percentage of each nucleus occurring in successive $30-\mu \mathrm{m}$ increments along the LS cord; number of labeled cells per nucleus also shown. stages 29 to 39 embryos with dorsoventrally rotated limbs, and, for comparison, eight stage 28 to 30 control embryos.

In four stage 29 experimental embryos in which the ventral shank was injected with HRP, and in four different stage 29 to 30 experimental embryos in which motoneurons innervating the dorsal shank were localized, the position of motoneurons within the spinal cord was appropriate in both the rostrocaudal and mediolateral planes. Occasionally, as was found in the older embryos, a segment which would normally innervate a muscle mass did not do so in the dorsoventrally rotated limb. However, all motoneurons that did innervate a muscle mass were appropriate. The histograms of Figure $8 \mathrm{com}$ bine the segmental distribution of motoneurons innervating four shank ventral (Fig. $8 A$ ) and four dorsal muscle masses (Fig. $8 B$ ) in embryos with dorsoventrally rotated limbs. It can be seen that the distribution of motoneurons is similar to the control distributions at this stage, also shown. The small number of motoneurons (2\%) from segment 3 which normally innervate the ventral shank did not appear to innervate the ventral shank in these dorsal-ventral limb rotations.

In Figures 9 and 10, HRP fills of one control muscle mass and the opposite muscle mass in an embryo with a dorsal-ventral limb rotation are combined in order for the reader to bettcr assess the degree of overlap in motoneuron distributions. Figure 9 shows an example of the mediolateral positions of motoneurons innervating the dorsal shank in one control embryo and the ventral shank in one embryo with a dorsal-ventral limb rotation. It can be seen that the distributions of motoneurons are fairly separate in the mediolateral axis between these embryos. Figure 10 shows an example of the mediolateral positions of motoneurons innervating the ventral shank in a control and the dorsal shank in an experimental embryo. Again, they are fairly separate, with little overlap in the distributions of motoneurons projecting to the ventral and dorsal shanks in either control or experimental embryos.

Axon palhways following dorsal-ventral rotation of the limb. To determine how motoneuron axons reached their correct target under these experimental conditions, I reconstructed the innervation pattern in stage 29 to 31 dorsoventrally rotated limbs. In those embryos in which axons to the injected muscle mass stained diffusely with $\mathrm{HRP}$, the paths of axons projecting to a single muscle or muscle mass could be followed from the spinal cord to the muscle they innervated.

In all eight embryos in which the motor nuclei were localized prior to motoneuron death, axon pathways were reconstructed. Thus, both the identity of the stained motoneurons and their pattern of growth through the periphery were known. For an additional eight embryos in which the nerve-branching pattern was reconstructed, the motoneuron locations were not known.

Axons compensate for the dorsal-ventral limb rotation by rearranging within the plexus and proximal nerve trunk. The insets in Figures 11 and 12 are camera lucida drawings of transverse sections of spinal and muscle nerves and indicate the changes in position of the stained axons as they grow through the limb. These can be 
A

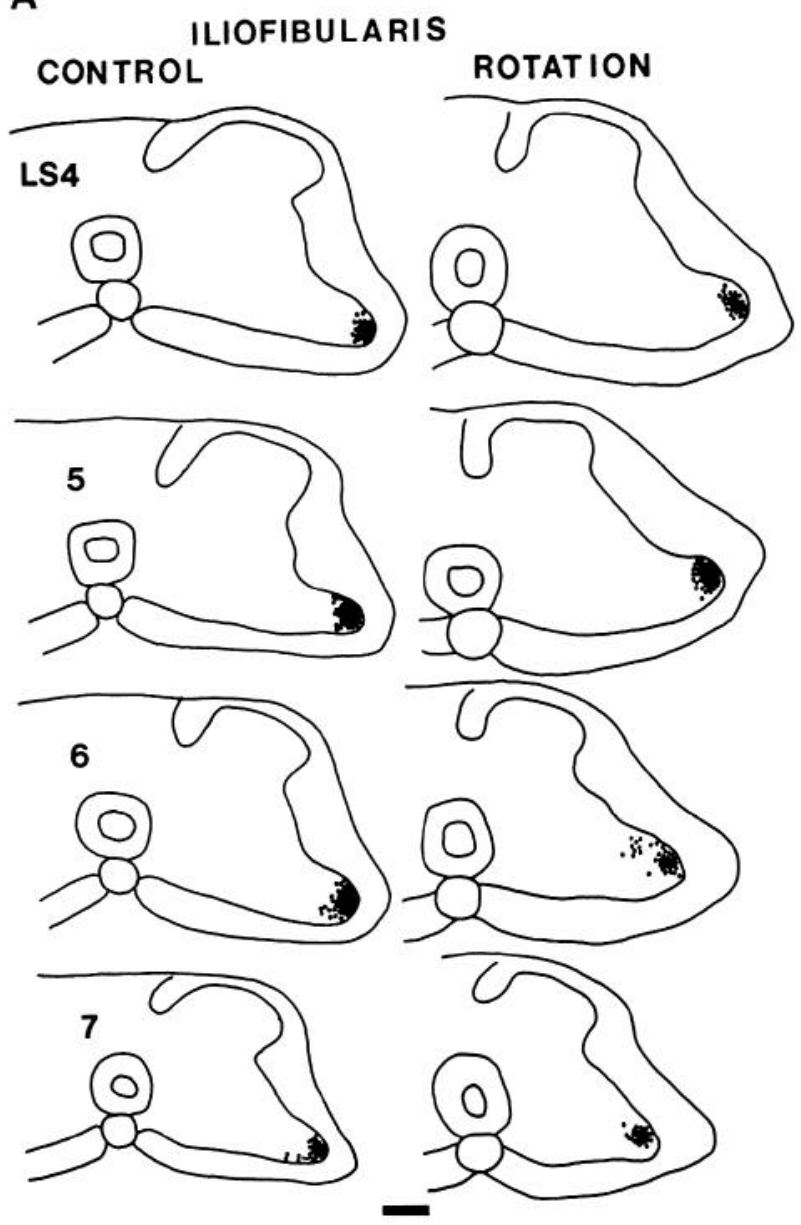

B

\section{Iliofibularis}

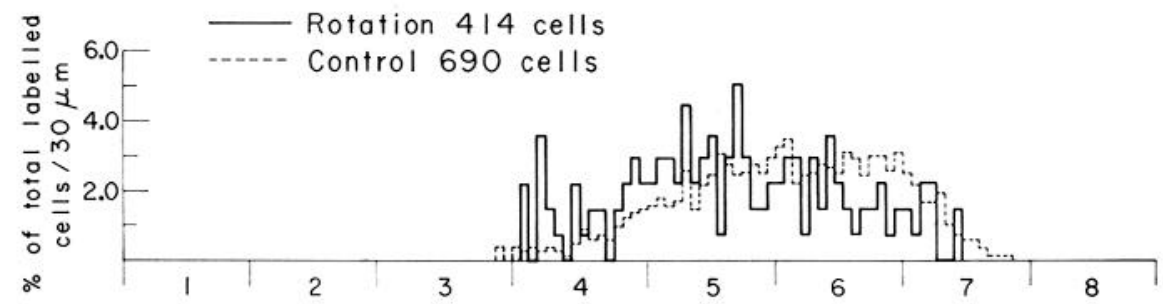

Figure 7. A, Transverse sections showing stained motoneurons following retrograde injections of HRP into the iliofibularis muscle in control (left) and experimental (right) embryos at stage 36. Each cross-section pools all stained motoneurons in every third $10-\mu \mathrm{m}$ section within a LS segment. Each stained motoneuron indicated by a dot. Calibration bar, $100 \mu \mathrm{m}$. B, Rostrocaudal position of motoneurons innervating the iliofibularis muscle in stage 36 control and experimental embryos. Histograms show the percentage of each nucleus occurring in successive $30-\mu \mathrm{m}$ increments along the LS cord, the number of labeled cells per nucleus is also shown.

compared to the insets of control reconstructions in Figure 1.

The example shown in Figure 11 demonstrates this rearrangement. Muscles of the dorsal shank were injected with HRP; axons to the dorsal shank in a control situation collect and remain in a lateral position in the nerve trunk. In the rotated limb, however, the axons initially converge and cluster within the nerve in a lateral position (Fig. 11, inset $C$ ) but subsequently compensate for the dorsal-ventral limb rotation by shifting to a medial position in the nerve (Fig. 11, inset $E$ ). This position is appropriate to reach the dorsal shank when the limb is dorsoventrally rotated. Thus, axons aggregate, then compensate for limb rotations by shifting position within a 


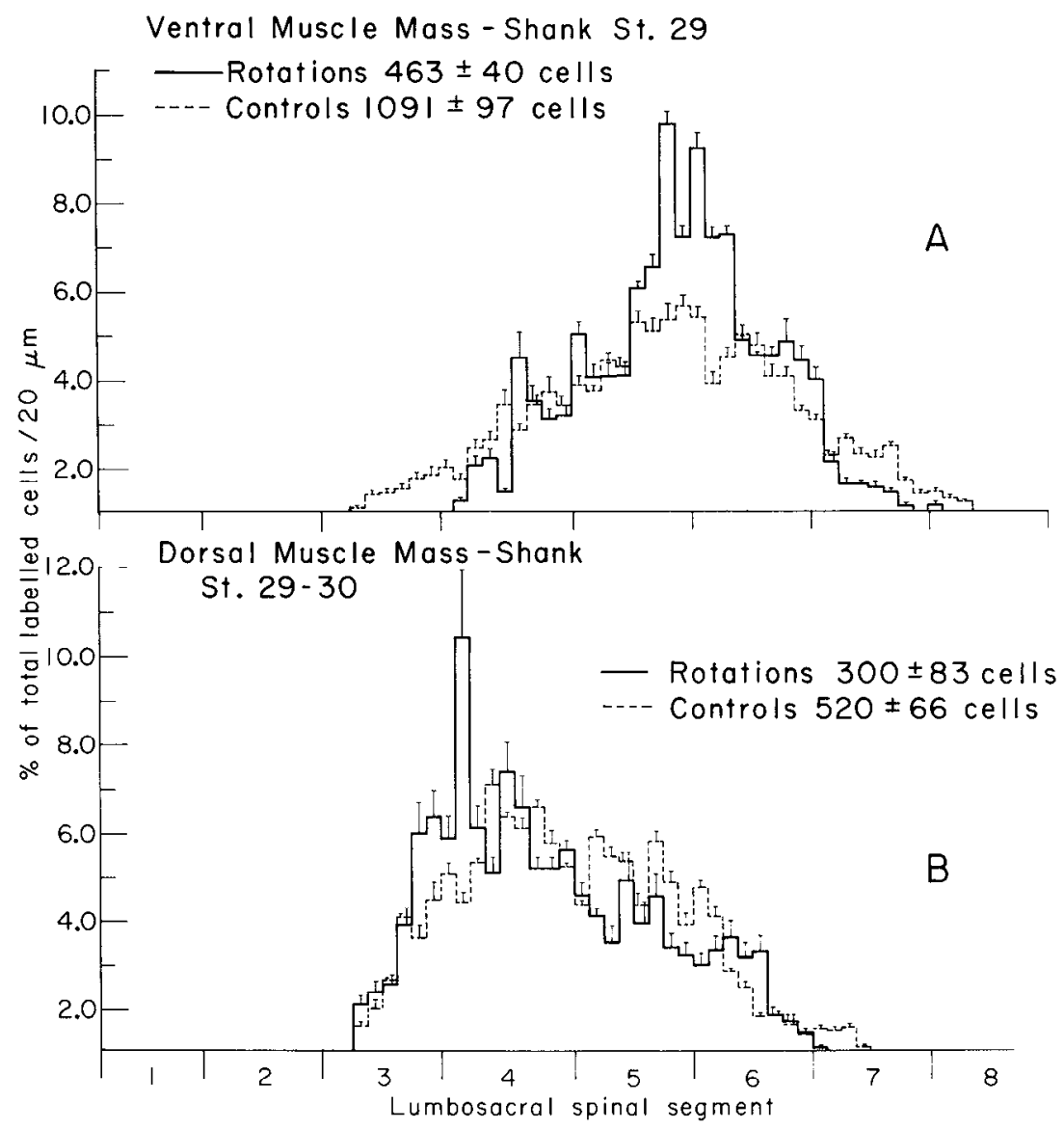

Figure 8. Rostrocaudal position of motoneurons innervating the shank ventral muscle mass $(A)$ and shank dorsal muscle mass $(B)$ in control and experimental embryos at stages 29 to 30 . Each histogram pooled from four embryos shows the mean percentage of each nucleus occurring in successive $20-\mu \mathrm{m}$ increments along the cord. The mean number of labeled cells per nucleus $\pm \mathrm{SE}$ is also shown.

nerve at a point which, at the time the reconstructions are made, is a relatively large distance from their targets.

An example of the rearrangement of axons across the dorsal-ventral axis in a rotated limb during growth to the ventral shank is shown in Figure 12. Stained and unstained axons again exit intermingled (Fig. 12, inset $A$ ), initially collecting relatively medially with respect to the body (Fig. 12, inset $B$ ). The axons then shift to a lateral position, appropriate within the dorsoventrally rotated limb to innervate the ventral shank (Fig. 12, insets $C$ and $D$ ). In this case, the stained axons do not collect into a tight cluster until the point in their outgrowth where they shift their dorsal-ventral position within the limb.

In some experiments, spinal nerve 1 or 8 did not enter the limb, but ended in the body wall or tail due to unintentional variations in the placement of the limb in the anterior-posterior axis. In addition, in two cases, possibly a result of physical constraints on the nerve pathway due to a change in the position of the femur, the portion of the crural plexus which innervated the dorsal thigh grew posterior to instead of anterior to the femur (e.g., LS segments 1 to 3 of Fig. 12).

\section{Discussion}

Axon pathways in control embryos. Since motoneurons innervating the dorsal and ventral shank are intermin- gled for some distance upon exiting the spinal cord (Fig. 1 , insets $A$ ), it is difficult to see how mechanical channeling of axons or the simple maintenance of topographic order among them could result in the aggregation of axons seen.

This study (Fig. 1) and that of Lance-Jones and Landmesser (1981) show that motoneurons that innervate the same periphery do not exit the spinal cord together; they exit the cord in a distributed fashion and cross other fibers to collect together at some distance from the cord. This crossing of axons occurs early in development, prior to motoneuron cell death and prior to morphogenetic events in the limb. Thus, motoneuron growth into the periphery is not by the parallel outgrowth of motoneuron axons whose positions within the nerve are related to the positions of their cell bodies. Some other position-related feature of a motoneuron, either birth date or somal location, may cause the regrouping of axons, possibly due to differences in adhesiveness of their growing tips, with like neurons tending to adhere to each other. While such mechanisms can explain the sorting out of axons destined for dorsal and ventral muscle masses which is observed in normal development, they cannot account for the ability of medial and lateral motoneurons to project to their original muscle masses following physical displacement of the target.

Axon pathways after dorsal-ventral limb rotation. What 


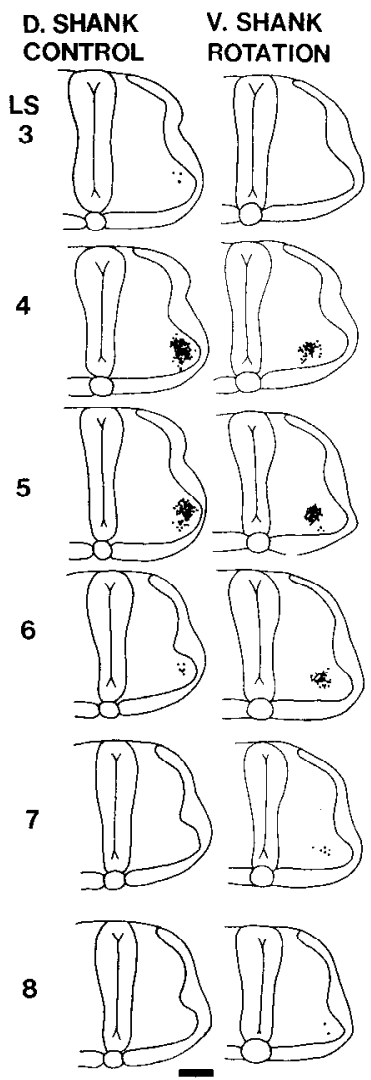

Figure 9. Transverse sections showing stained motoneurons following injections of HRP into the shank dorsal muscles mass at stage 29 in a control embryo (left) and the shank ventral muscle mass in an experimental embryo (right). HRP fills are presented in this manner to better assess the degree of separation between motoneuron distributions projecting to the ventral and dorsal muscle masses. Each cross-section combines all stained motoneurons in every second $10-\mu \mathrm{m}$ section within a LS segment. Each stained motoneuron is indicated by a dot. Calibration bar, $100 \mu \mathrm{m}$.

mechanisms could account for the results? Prior to entering the limb, axons could recognize the altered limb orientation and respond by rearranging themselves along the dorsal-ventral axis. Subsequent growth through the limb could then occur with the same branching pattern as normal, but reversed in a mirror image way with respect to the body axis.

Reconstruction of axon pathways in experimental embryos (Figs. 11 and 12) showed that motoneuron axons indeed compensated for limb rotation by altering position within the plexus and proximal nerve trunk in order to project to their normal targets. A comparison of the projection patterns in the experimental embryos with the control projections shown in Figure 1 shows that the nerve-branching patterns to the muscles are basically the same. Axons compensate for the dorsal-ventral limb rotation by shifting relative positions within the plexus, not by forming abnormal nerves or taking abnormal routes in the regions of their targets. The cues used by the axons to sort into positions appropriate for their targets were detected before axons reached their targets. What the distance actually is between an axon and its target at the point at which an axon makes this path choice is not known.
In a few cases there are abnormalities in the pattern of nerve projection after limb rotation. For example, in the experiment of Figure 11, spinal nerves 7 and 8 do not join the sciatic plexus as normal, but do grow together into the limb, branch normally, and innervate appropriate muscles. In Figure 12, axons from spinal nerves 1 to 3 grow posterior to instead of anterior to the femur. These differences could be due to conflicting positional cues as axons pass from unrotated (body wall) to rotated (limb) tissue, and/or slight shifts of the limb in the anterior-posterior direction.

Comparison to other experimental manipulations. Even in experimental situations in which motoneurons innervate inappropriate muscles (for example, upon addition of supernumerary limbs), the motoneurons, although from inappropriate rostrocaudal lumbosacral levels, are found in the appropriate mediolateral position in the spinal cord (chick: Hollyday et al., 1977; Morris, 1978; Hollyday, 1981; Landmesser and Lance-Jones, 1981; Xenopus: Rubin and Mendell, 1980). Since axon sorting along the dorsal-ventral limb axis occurs even when the motoneurons are from a position inappropriate for the ultimate target, it may be that the cues responsible for the aggregation of axons destined for dorsal and ventral musculature separate axons into two general classes (medial and lateral motoneurons) and not numerous sub-

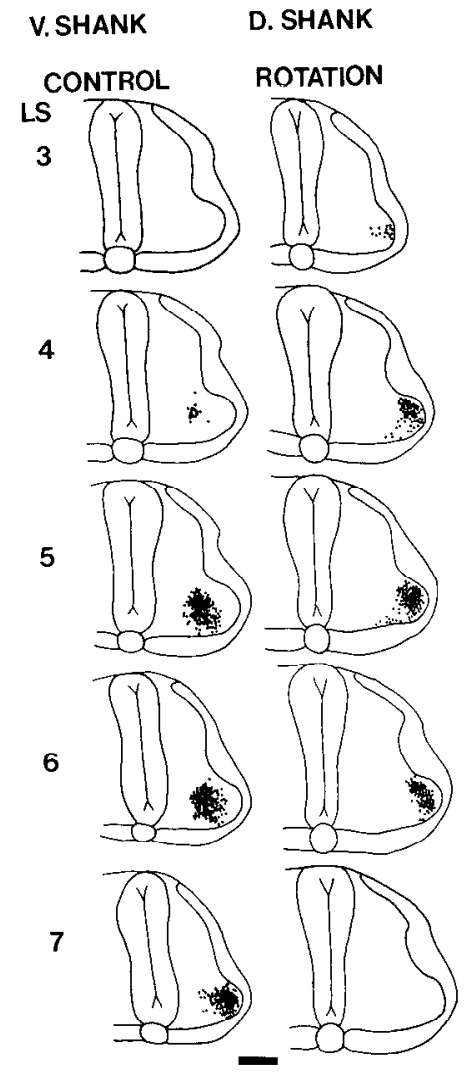

Figure 10. Transverse sections showing stained motoneurons following injections of HRP into the shank ventral muscle mass at stage 29 in control (left) and dorsal muscle mass in an experimental (right) embryo. Each cross-section pools all stained motoneurons in every second $10-\mu \mathrm{m}$ section within a LS segment. Each stained motoneuron is indicated by a dot. Calibration bar, $100 \mu \mathrm{m}$. 


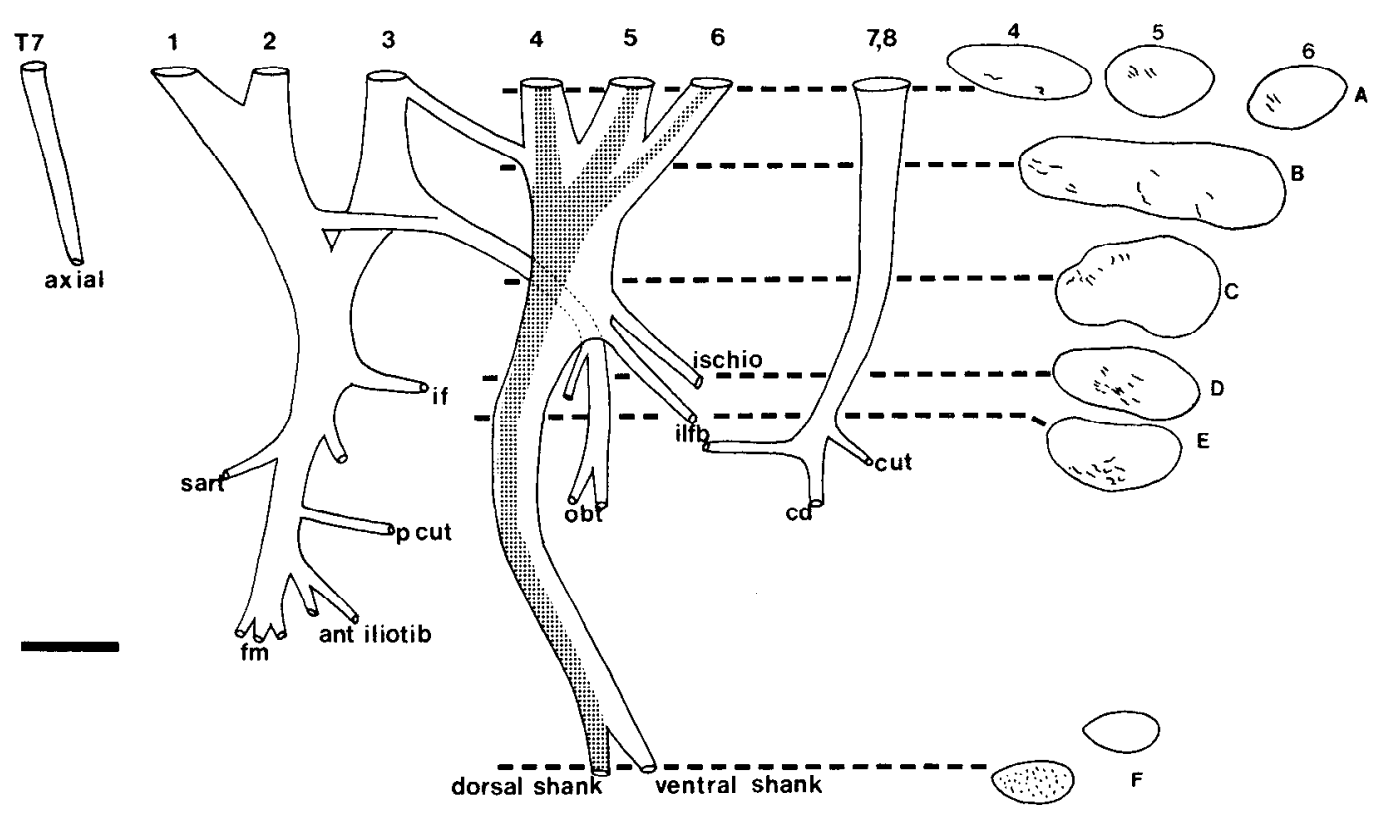

Figures 11 and 12. Reconstructions of nerve patterns in the hindlimb at stages 29 to 30 following dorsal-ventral limb bud rotation at stages 16 to 17 . Pathways through the limb of axons labeled by retrograde uptake of HRP injected into either the shank dorsal (Fig. 11) or ventral (Fig. 12) masses are shown by stippling. Insets $(A$ to $F$ ) indicate the positions of stained axons within the nerves at selected positions in their outgrowth to the periphery; lateral is up, medial is down. sart, sartorius; fm, femorotibialis; ant iliotib; anterior iliotibialis; obt, obturator; $p$ iliotib, posterior iliotibialis; ischio, ischioflexorius; $c d$, caudilioflexorius; ilfb, iliofibularis; $p$ cut, posterior cutaneous; if, iliofemoralis; cut, anterior cutaneous.

Figure 11. Stained axons to the shank dorsal muscle mass exit the spinal cord intermingled with unstained axons $(A)$, collect laterally (up) as shown in $C$, and then shift, compensating for the dorsal-ventral limb rotation, to a medial (down) position shown in $E$.

classes (for example, individually by motor pools). This aggregation may be an interaction among axon tips themselves or may be independent responses by each axon to the limb orientation. Studies on the fasciculation behavior of identified axons during growth in tissue culture may resolve some of these questions.

Whenever either appropriate or entirely inappropriate axons are allowed to innervate the limb, after cord rotations, limb rotations, limb shifts, limb transplantations to ectopic sites, and addition of supernumerary limbs (Harrison, 1908; Detwiler, 1920; Hamburger, 1939; Piatt, 1956; Morris, 1978; Lance-Jones and Landmesser, 1980; Hollyday, 1981; Landmesser and Lance-Jones, 1981; this paper), the plexus with crural and ischiadic nerve trunks forms relatively normally. Thus inappropriate axons can trace out the same general routes through the limb as can appropriate axons. At the point in axon outgrowth at which the plexus is formed and at positions of major nerve divergence within the limb, strong environmental constraints, either of a mechanical nature or due to the presence of chemical cues, must exist dictating convergence and divergence of axons.

The results reported here support the conclusions of Lance-Jones and Landmesser (Lance-Jones and Landmesser, 1980; Landmesser and Lance-Jones, 1981) following manipulations of the spinal cord and limb in the chick. After rotations of small sections of the spinal cord (Lance-Jones and Landmesser, 1980) motoneurons compensated for the change in their point of entry into the limb by altering position within the plexus or major nerve trunks in order to innervate their appropriate target.
Equivalent results were found before and after cell death. Thus, in addition to the specification of motoneuron destinations along the dorsal-ventral axis seen in the limb rotation experiments, their experiment shows that motoneurons are specified prior to outgrowth for a particular peripheral termination along the anterior-posterior axis and are capable of detecting and compensating for changes in their position along this axis.

My data are consistent with data from the experiments discussed above, but are in apparent conflict with those of Summerbell and Stirling (1981). Upon dorsal-ventral rotation of the chick wing, motoneurons in only a minority of cases innervated their appropriate targets. In the majority of cases they innervated an inappropriate target. 'Their operations were done at later stages than in the experiments reported here (stages 18 to 20 versus stages 16 to 17) and thus the level of reversal was further distal (near the elbow or shoulder in contrast to the limb base). Since, before reaching the elbow, axons destined to innervate dorsal and ventral limb musculature are already separated into nerves, a rotation at this level may present a situation which is too extreme for the axons to compensate for by altered growth. The axons may either detect the change yet be physically incapable of compensating, or not be able to detect the position of its appropriate target and so form nonspecific synapses.

Number of labeled motoneurons. In control embryos, fewer motoneurons label with HRP prior to cell death than after, even though more motoneurons are present (histograms of Figs. 5 and 8). This could be due to the fact that some motoneuron axons may not yet have 


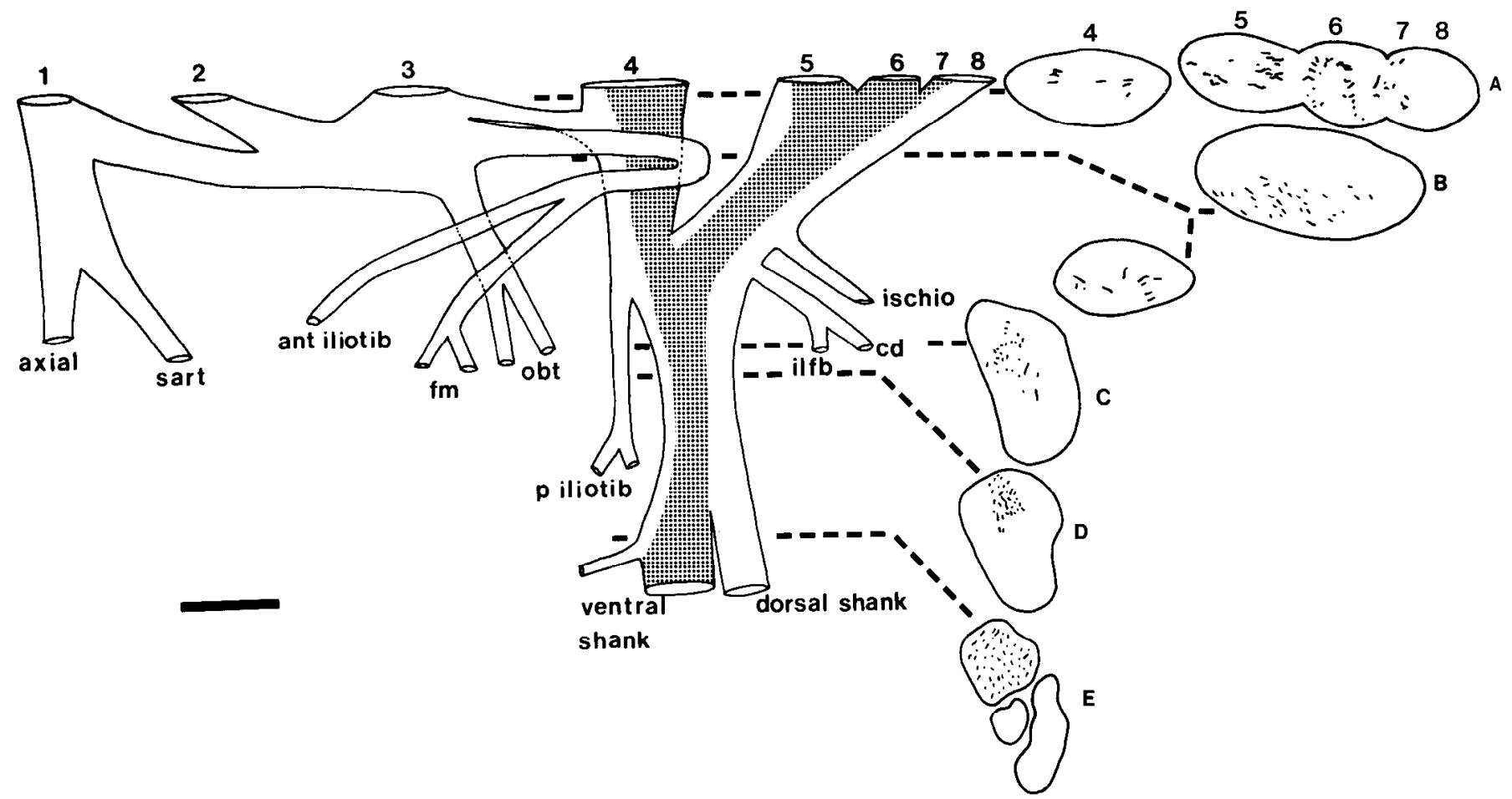

Figure 12. Stained axons to the shank ventral muscle mass exit intermingled with unstained axons $(A)$, collect medially (down) in $B$, and then shift $(C)$ to a lateral (up) position in D. Calibration bar, $200 \mu \mathrm{m}$.

ramified within the muscle mass by stages 29 to 30 , or that all motoneuron axons may not pick up HRP at the early stages (Landmesser, 1978b).

In addition, the number of labeled motoneurons is lower in experimentals as compared to controls, both before and after cell death (Figs. 3 to 10). The number of labeled motoneurons varies depending on the size of HRP injections, which was usually smaller in experimental embryos so as to prevent leakage of the enzyme. However, this difference cannot account fully for the discrepancy in the number of labeled motoneurons.

Prior to motoneuron death, only 12 to $58 \%$ of the control number of labeled motoneurons innervating the shank ventral or dorsal muscle mass were labeled in embryos with rotated limbs. This is not due to a lower number of motoneurons in experimental embryos. Comparison of the total number of motoneurons in LS4 between the control and experimental sides of four stage 28 to 29 embryos showed a 1 to $4 \%$ difference in the counts, not consistently lower on either side. The lower number of labeled motoneurons prior to motoneuron death could be due to fewer axons entering the limb; reconstructions of axon pathways in young embryos show fiber bundles which did not enter the limb but entered the body wall, possibly due to a confusion of positional cues at the junction between body and rotated limb. In addition, there could be less extensive branching of terminals within the limb, perhaps from a slightly smaller limb or a slowing of development as a result of the operation. One might argue that terminals in rotated limbs do not pick up HRP for some reason, although this is unlikely to be a result of the presence of inactive terminals, as blocking neuromuscular function does not block uptake of HRP using this technique (Oppenheim, 1981).

After the period of motoneuron death, 28 to $60 \%$ of the control number of shank-innervating motoneurons were labeled with HRP in experimental embryos. In these cases, counts show that the total number of motoneurons was lower on the experimental side, different in each embryo. The lower number of motoneurons after the period of cell death is probably due to the death of those motoneurons that do not enter the limb. Those motoneurons which did enter the limb made appropriate connections.

In summary, motoneuron axons can detect and compensate for the dorsal-ventral rotation of the limb bud in order to innervate their appropriate targets. Motoneuron axons compensate by altering growth within the plexus and nerve trunk prior to reaching the muscles in order to be in an appropriate position to innervate their targets. This shift in axon positions results in a pattern of limb innervation that is mirror image reversed to normal across the dorsal-ventral body axis; thus the pattern is normal within the dorsoventrally reversed limb.

Thus it seems that growing axons must utilize specific cues, possibly chemical, existing regionally within the limb in their growth through the limb. The cues could be of a general nature, simply segregating axons across the dorsal-ventral limb axis into two groups; or the dorsalventral separation of axons could be the individual responses of several subgroups of axons to environmental cue(s). However many groups of axons exist, the motoneurons must differ from each other in a way that allows them to recognize the dorsal-ventral limb axis and re- 
spond differentially to changes in its orientation during outgrowth.

\section{References}

Bixby, J. L., J. H. R. Maunsell, and D. C. Van Essen (1980) Effects of motor unit size on innervation patterns in neonatal mammals. Exp. Neurol. 70: 516-524.

Cruce, W. L. R. (1974) The anatomical organization of hindlimb motoneurons in the spinal cord of the frog, $R$. catesbiana. J. Comp. Neurol. 153: 59-76.

Detwiler, S. R. (1920) Experiments on the transplantation of limbs in Amblystoma. The formation of nerve plexuses and the function of the limbs. J. Exp. Zool. 31: 117-169.

Hamburger, V. (1939) The development and innervation of transplanted limb primordia of chick embryos. J. Exp. Zool. 80: $347-385$

Hamburger, V. (1975) Cell death in the development of the lateral motor column of the chick embryo. J. Comp. Neurol. 160: $535-546$.

Hamburger, V., and H. L. Hamilton (1951) A series of normal stages in the development of the chick embryo. J. Morphol. 88: 4992.

Harrison, R. G. (1908) Embryonic transplantation and development of the nervous system. Anat. Rec. 2: 367-410.

Hollyday, M. (1980) Organization of motor pools in the chick lumbar lateral motor column. J. Comp. Neurol. 194: 143-170.

Hollyday, M. (1981) Rules of motor innervation in chick embryos with supernumerary limbs. J. Comp. Neurol. 202: 439465.

Hollyday, M., and V. Hamburger (1977) An autoradiographic study of the formation of the lateral motor column in the chick embryo. Brain Res. 132: 197-208.

Hollyday, M., V. Hamburger, and J. M. G. Farris (1977) Localization of motor pools supplying identified muscles in normal and supernumerary legs of chick embryo. Proc. Natl. Acad. Sci. U. S. A. 74: 3582-3586.

Horder, T. J. (1978) Functional adaptability and morphogenetic opportunitism, the only rules for limb development? Zoon 6 : 181-192.

Lance-Jones, C., and L. Landmesser (1980) Motoneurone projection patterns in the chick hind limb following early partial reversal of the spinal cord. J. Physiol. (Lond.) 302: 581-602.

Lance-Jones, C., and L. Landmesser (1981) Pathway selection by chick lumbosacral motoneurons during normal development. Proc. R. Soc. Lond. Biol. 214: 1-18.

Landmesser, L. (1978a) The distribution of motoneurons supplying chick hind limb muscles. J. Physiol. (Lond.) 284: 371389.

Landmesser, L. (1978b) The development of motor projection patterns in the chick hind limb. J. Physiol. (Lond.) 284: 391414.

Landmesser, L., and D. G. Morris (1975) The development of functional innervation in the hind limb of the chick embryo. J. Physiol. (Lond.) 249: 301-326.

Landmesser, L. T., and C. Lance-Jones (1981) An experimental analysis of pathway selection by lumbosacral motoneurons in the chick embryo. In Development of the Nervous System,
D. Garrod and J. D. Feldman, eds., pp. 215-231, Cambridge University Press, London.

Letourneau, P. C. (1975a) Possible roles for cell to substratum adhesion in neuronal morphogenesis. Dev. Biol. 44: 77-91.

Letourneau, P. C. (1975b) Cell to substratum adhesions and guidance of axonal elongation. Dev. Biol. 44: 92-101.

Ludueña, M. A., and N. K. Wessels (1973) Cell locomotion, nerve elongation and microfilaments. Dev. Biol. 30: 427-440.

Lyser, K. M. (1971) Microtubules and filaments in developing axons and optic stalk cells. Tissue Cell 3: 395-404.

Morris, D. G. (1978) Development of functional motor innervation in supernumerary hind limbs of the chick embryo. J. Neurophysiol. 41: 1450-1465.

Oppenheim, R. W. (1981) Cell death of motoneurons in the chick embryo spinal cord. V. Evidence on the role of cell death and neuromuscular function in the formation of specific peripheral connections. J. Neurosci. 1: 141-151.

Piatt, J. (1956) Studies on the problem of nerve pattern. I. Transplantation of the forelimb primordium to ectopic sites in Amblystoma. J. Exp. Zool. 131: 173-201.

Rakic, P. (1971) Neuron-glia relationship during granule cell migration in developing cerebellar cortex. A Golgi and electron microscopic study in Macacus rhesus. J. Comp. Neurol. 141: $282-312$

Romanes, C. (1951) The motor cell columns of the lumbosacral spinal cord of the cat. J. Comp. Neurol. 94: 313-364.

Romanes, C. (1961) The motor pools of the spinal cord. Prog. Brain Res. 11: 93-119.

Romer, A. (1927) The development of the thigh musculature of the chick. J. Morphol. 43: 347-385.

Rubin, D. I., and L. M. Mendell (1980) Location of motoneurons supplying individual muscles in normal and grafted supernumerary limbs of Xenopus laevis. J. Comp. Neurol. 192: $703-715$.

Sharrard, W. J. W. (1955) The distribution of the permanent paralysis in the lower limb in poliomyelitis. J. Bone Joint Surg. Am. 37B: 540-558.

Silver, J., and R. M. Robb (1979) Studies on the development of the eye cup and optic nerve in normal mice and mutants with congenital optic nerve aplasia. Dev. Biol. 68: 175-190.

Silver, J., and R. L. Sidman (1980) A mechanism for the guidance and topographic patterning of retinal ganglion cell axons. J. Comp. Neurol. 189: 101-111.

Sperry, R. W. (1963) Chemoaffinity in the orderly growth of nerve fiber patterns and connections. Proc. Natl. Acad. Sci. U. S. A. 50: 703-710.

Suburo, A., N. Carri, and R. Adler (1979) The environment of axonal migration in the developing chick retina. A scanning electron microscope (SEM) study. J. Comp. Neurol. 184:519536 .

Summerbell, D., and R. V. Stirling (1981) The innervation of dorsoventrally reversed chick wings: Evidence that motor axons do not actively seek out their appropriate targets. J. Embryol. Exp. Morphol. 61: 233-247.

Wortham, R. A. (1948) The development of the muscles and tendons in the lower leg and foot of chick embryos. J. Morphol. 83: 105-148. 